\title{
Improved Equivalent Circuit and Analytical Model for Amorphous Silicon Solar Cells and Modules
}

\author{
J. Merten, J. M. Asensi, C. Voz, A. V. Shah, R. Platz, and J. Andreu
}

\begin{abstract}
An improved equivalent circuit for hydrogenated amorphous silicon $(a-\mathrm{Si}: \mathrm{H})$ solar cells and modules is presented. It is based on the classic combination of a diode with an exponential current-voltage characteristic, of a photocurrent source plus a new term representing additional recombination losses in the $i$ layer of the device. This model/equivalent circuit matches the $I(V)$ curves of $a$-Si:H cells over an illumination range of six orders of magnitude. The model clearly separates effects related to the technology of the device (series and parallel resistance) and effects related to the physics of the pin-junction (recombination losses). It also allows an effective $\mu \tau$ product in the i-layer of the device to be determined, characterizing its state of degradation.
\end{abstract}

Index Terms - Amorphous silicon solar cells and modules, analytical model, $I(V)$ characteristics, outdoor measurements, recombination, $\mu \tau$-product degradation.

\section{INTRODUCTION}

$\mathbf{T}$ HE use of equivalent circuits is a convenient and common way to describe the electrical behavior of electronic devices. Generally, an equivalent circuit offers three main advantages: it is easy to use within electrical circuits; it allows the device's properties to be described in a standardized and abbreviated manner using a simple analytical model; it provides insights into the complex physical processes that take place within the device.

The equivalent circuit generally used for photovoltaic solar cells is shown in Fig. 1 (ignoring the dashed section): it essentially consists of a current source shunted by a diode. These two elements correspond to generation and loss of photocurrent in the device. The resistances $R_{s}$ and $R_{p}$ can be considered to be "parasitic" circuit elements, introduced to describe the behavior of real solar cells with their technical limitations. We shall come back to these later.

That one may simply superpose a photocurrent source $I_{\mathrm{ph}}$ on the characteristics of the dark diode is, at first sight, physically surprising - in fact, the photo-induced generation of holes and electrons within the solar cell will change the carrier concentration at every point, requiring, thus, a new solution for the drift-diffusion differential equations throughout the

Manuscript received October 10, 1996; revised July 10, 1997. The review of this paper was arranged by Editor P. N. Panayotatos. This work was supported by the EU Project JOU2-CT94-0403, Project EF-REN (93)032 of the Swiss Confederation, by the Spanish Goverment, and the Generalitat de Catalunya.

J. Merten, J. M. Asensi, C. Voz, and J. Andreu are with Universidad de Barcelona, Departament de Física Aplicada i Eletrònica, E-08028 Barcelona, Spain.

A. V. Shah and R. Platz are with Institut de Microtechnique, Rue Breguet, Switzerland.

Publisher Item Identifier S 0018-9383(98)00969-1.

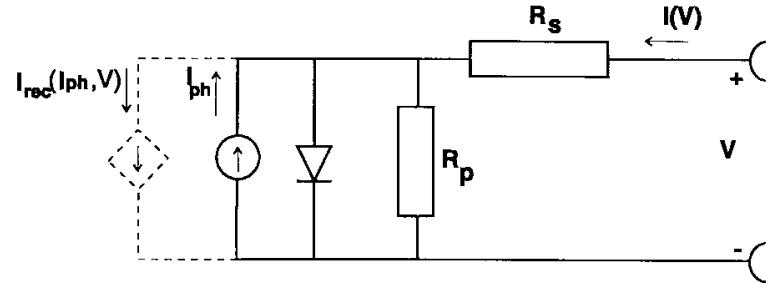

Fig. 1. Equivalent circuit for photovoltaic solar cells and modules. The current sink (dashed lines) takes into account the current losses due to recombination in the i-layer of the device.

whole device. However, as postulated in [1], and as shown in [2], such a simple superposition of a dark diode and a photocurrent source is indeed valid and can be theoretically justified for crystalline solar cells, consisting of pn-diodes. The theoretical justification [2] is based on the linear form of the drift-diffusion differential equations for minority carriers, within the p- or n-type bulk regions of the pn-diode.

It is, however, well known that amorphous silicon solar cells, which are pin-diodes and in which the main part of the photovoltaic generation occurs in the intrinsic i-layer, behave differently. As a striking example, the $I(V)$ curves for different illumination levels usually all meet at a single point $I_{C}\left(V_{C}\right)$ in the first quadrant [3], a fact that can only be reconciled with the simple equivalent circuit of Fig. 1 if an additional loss term, which increases strongly with the forward voltage $V$, is introduced. Such a loss term has to take into account the recombination losses in the intrinsic layer of the device.

Generally speaking, recombination is relatively intense within amorphous silicon cells because of the presence of dangling bonds that act as recombination centers-this is especially true for cells in the degraded state. It is therefore intuitively "reasonable" to describe amorphous silicon cells by introducing an additional recombination loss term $I_{\text {rec }}$ into the equivalent circuit, a term which is symbolized by the current sink (dashed lines) in Fig. 1. Recombination losses within the i-layer are in a first approximation proportional to the carrier concentrations observed therein, and thus to the photogenerated current $I_{\mathrm{ph}}$. This has been shown theoretically in [4] and is used in the formulation of the loss current $I_{\text {rec }}$.

The aims of this article are fourfold:

1) to show empirically that the equivalent circuit in Fig. 1 describes quite precisely the experimentally measured electrical behavior of illuminated solar cells and is able to do so for illumination levels varying over six orders of magnitude; 


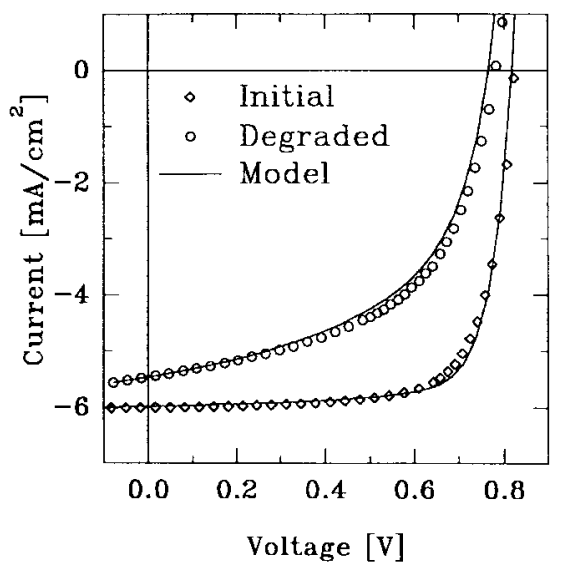

Fig. 2. Comparing the experimental $I(V)$-data of the laboratory cell shown in Fig. 3 with the results of the analytical model. The decrease of $R_{\mathrm{Sc}}=(\partial V / \partial I)_{V=0}$ of the cell in the degraded state is attributed to enhanced recombination in the i-layer of the device. Note that the VIM-method does not require calibrated illumination levels, these may therefor differ for the measurement of the cell in the degraded and initial state.

2) to present an experimental method that can be used to determine systematically the elements of this equivalent circuit, including the new recombination loss term (dashed symbol in Fig. 1);

3) to provide a link between the equivalent circuit of Fig. 1, especially between the newly introduced recombination loss term, and theoretical treatments of recombination in amorphous silicon cells, such as [4];

4) and to illustrate the use of the new, complete equivalent circuit to investigate the long-term behavior of a commercial module during outdoor exposition.

\section{EXPERIMENTAL PROCEDURE}

The authors have used the standard characterization procedure for solar cells that consists of measuring the $I(V)$ curve at a given illumination level as it shown in Fig. 2. The resulting data may be condensed into the six characteristic parameters which are:

1) the short circuit current $I_{\mathrm{sc}}$;

2) the open circuit voltage $V_{o c}$;

3) the fill factor $F F$;

4) the efficiency $\eta$;

5) the "open circuit resistance" $R_{\mathrm{oc}}=(\partial V / \partial I)_{I=0}$, which may be related to the series resistance $R_{s}$;

6) and the "short circuit resistance" $R_{\mathrm{sc}}=(\partial V / \partial I)_{V=0}$, which may be related to the parallel resistance $R_{p}$.

The latter two parameters $R_{\mathrm{oc}}$ and $R_{\mathrm{sc}}$ are key parameters for the present treatment; they are reciprocal slopes of the $I(V)$ curve.

The basic idea is to measure the $I(V)$ curve over a wide range of illumination levels, rather than at a fixed illumination level of say $1000 \mathrm{~W} / \mathrm{m}^{2}$. Thereby, additional information about the device can be gained. We call this method the Variable Illumination Measurement (VIM) method.

The experimental results are plotted as a function of the short circuit current $I_{\mathrm{sc}}$ or open circuit voltage $V_{\mathrm{oc}}$ avoiding the need of calibrated illumination levels. This has been done with the data obtained from a typical laboratory solar cell [5] (single junction, glass $/ \mathrm{SnO}_{2} /$ pin-structure with an i-layer thickness $d_{i}$ of $0.35-\mu \mathrm{m}, 10.7 \%$ initial efficiency) which are are indicated by the symbols in Fig. 3 .

The light source may be either a laboratory lamp or, alternaltively, sunlight for outdoor measurements. In the laboratory, variation of the illumination level over six orders of magnitude is obtained by varying the distance between the lamp and the sample and by using neutral (grey) filters. The outdoor VIM-method makes use of the natural variation of the solar irradiance, the spectral variations having only a slight influence on the results in the case of single junction cells.

\section{The Analytical Model}

The single exponential model represented by the equivalent circuit in Fig. 1 ignoring the dashed section is known to match well the $I(V)$ curves of crystalline solar cells [1], [2]. This model predicts that the short-circuit resistance $R_{\mathrm{SC}}$ should be equal to the parallel resistance $R_{p}$ of the device over a large range of illumination levels [dot-dashed line in Fig. 3(b)]. The experimental $R_{\mathrm{sc}}\left(I_{\mathrm{sc}}\right)$-data show that this is not the case for amorphous silicon cells. This constitutes a clear empirical motivation for the introduction of a new current loss term into the equivalent circuit (represented by the dashed section in Fig. 1): a term which explicitly takes into account the recombination losses in the i-layer of the device. A simple expression for this current can be deduced assuming the electrical field $|E|$ to be constant within the i-layer, and to be strong enough to mask the effects of the diffusion of the carriers [4]. This very crude assumption is expected to be valid only for small or negative external voltages $V$, for cells with thin i-layers (small value of $d_{i}$ ) and for low defect densities therein. In this case, a homogenous generation of carriers leads to linearily varying profiles for the free electrons $n_{f}$ and holes $p_{f}$ in the i-layer [4]. The recombination function is taken from [6]

$$
R_{D B}=\frac{n_{f}}{\tau_{n}^{0}}+\frac{p_{f}}{\tau_{p}^{0}}
$$

where $\tau_{n}^{0}$ and $\tau_{p}^{0}$ are the capture times of the electrons and holes by the neutral dangling bonds. Inserting the linear carrier profiles mentioned above, this recombination function becomes

$$
R_{D B}(x)=G \cdot\left[\frac{x}{d_{i}}+\frac{\mu_{n}^{0} \tau_{n}^{0}}{\mu_{p}^{0} \tau_{p}^{0}}\left(1-\frac{x}{d_{i}}\right)\right] \frac{d_{i}}{\mu_{n}^{0} \tau_{n}^{0}|E|}
$$

where $x$ is the position in the i-layer measured as the distance from the p-layer, $\mu_{n}^{0}$ and $\tau_{p}^{0}$ the band mobilities of the free carriers. Remember that this expression has been obtained neglecting diffusion currents and is only valid for strong fields in the i-layer, thin cells and low defect densities in the i-layer.

The expression for the recombination $R_{D B}(x)$ in (2) may be integrated over the whole i-layer (from $x=0$ to $x=d_{i}$ ) to obtain the total current loss $I_{\text {rec }}$ due to recombination within the i-layer; we thereby obtain

$$
I_{\mathrm{rec}}=I_{\mathrm{ph}} \cdot \frac{d_{i}}{(\mu \tau)_{\mathrm{eff}}\left[V_{\mathrm{bi}}-\left(V-I R_{s}\right)\right] / d_{i}}
$$




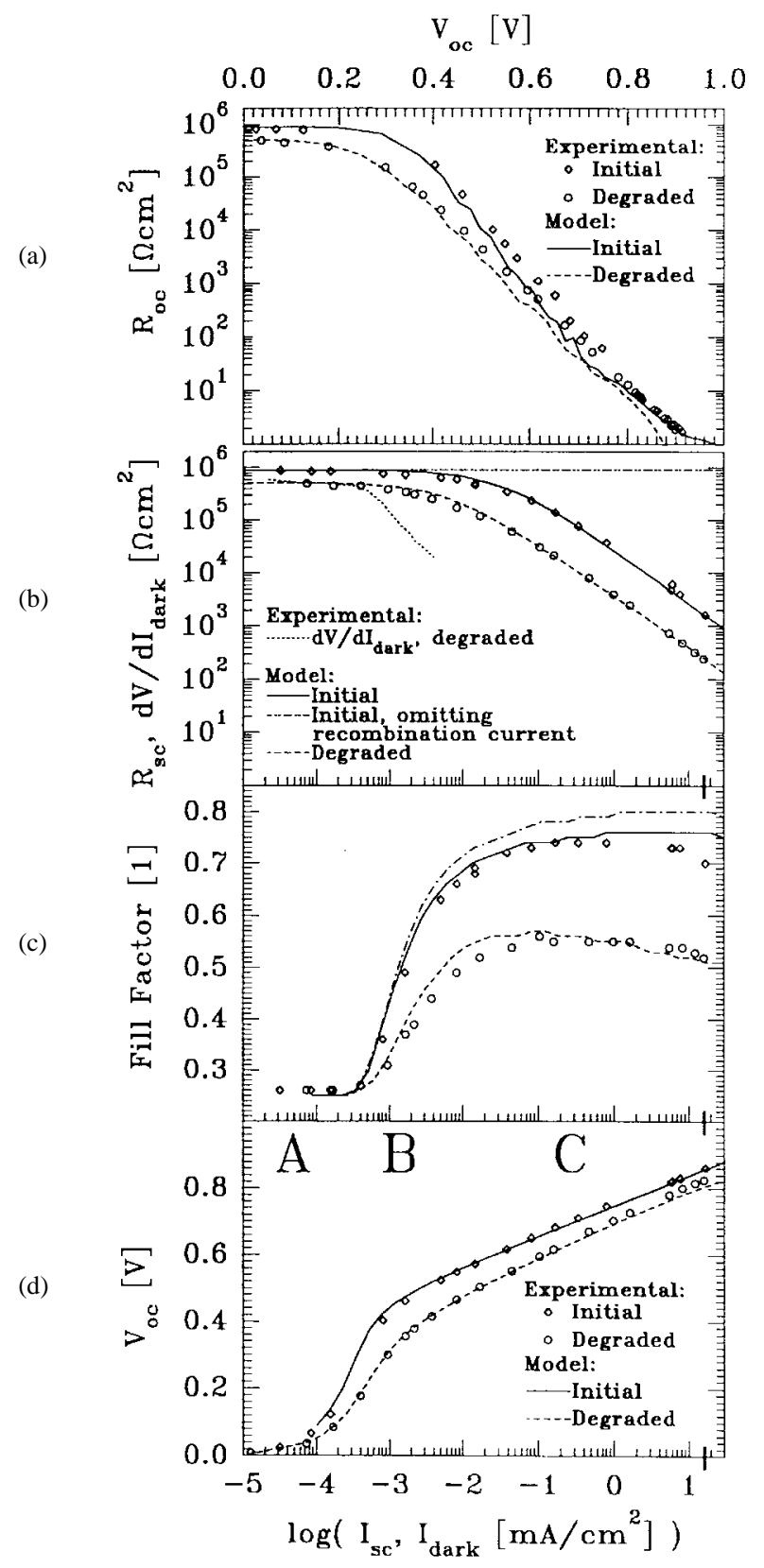

Fig. 3. Illumination level dependence of the $I(V)$ parameters. The symbols indicate the experimental data of a typical single junction laboratory cell, the lines the predictions of the model. Omitting the recombination current (dot-dashed lines) affects only the predictions for $R_{\mathrm{sc}}$ and $F F$, but not those for $V_{\mathrm{OC}}$ and $R_{\mathrm{OC}}$. The marks on the $x$-axis denote $I_{\mathrm{sc}}$ under one-sun illumination, and the dotted line $\left(\partial V / \partial I_{\text {dark }}\right)$ as a function of $I_{\text {dark }}$. The oscillations of $R_{\mathrm{OC}}$ are a numerical effect.

with

$$
(\mu \tau)_{\mathrm{eff}}=2 \frac{\mu_{n}^{0} \tau_{n}^{0} \cdot \mu_{p}^{0} \tau_{p}^{0}}{\mu_{n}^{0} \tau_{n}^{0}+\mu_{p}^{0} \tau_{p}^{0}}
$$

It should be noted that $I_{\text {rec }}$ equals the generation current $I_{\mathrm{ph}}$ multiplied by the ratio of the cell thickness $d_{i}$ over the effective driftlength $(\mu \tau)_{\text {eff }}|E|$ (Schubweg) in the i-layer. The electrical field $|E|$ in the i-layer (assumed to be constant) is set to the difference between the built-in voltage $V_{\mathrm{bi}}$ and the voltage over the junction $\left(V-I R_{s}\right)$. The effective $\mu \tau$-product
TABLE I

Parameter Values Used to Fit the Model to the Experimental Data of a Typical Laboratory Cell in the Degraded (B) and Initial (A) State

\begin{tabular}{c|c|c|c|c|c}
\hline & $\begin{array}{c}I_{0} \\
{\left[\mathrm{~mA} / \mathrm{cm}^{2}\right]}\end{array}$ & $\begin{array}{c}n \\
{[1]}\end{array}$ & $\begin{array}{c}R_{p} \\
{\left[\Omega \mathrm{cm}^{2}\right]}\end{array}$ & $\begin{array}{c}R_{s} \\
{\left[\Omega \mathrm{cm}^{2}\right]}\end{array}$ & $\begin{array}{c}\mu \tau \\
{\left[\mathrm{cm}^{2} / \mathrm{V}\right]}\end{array}$ \\
\hline $\mathrm{A}$ & $3.0 \cdot 10^{-8}$ & 1.6 & $9.0 \cdot 10^{5}$ & 1.0 & $4.7 \cdot 10^{-8}$ \\
\hline $\mathrm{B}$ & $5.0 \cdot 10^{-7}$ & 2.0 & $5.3 \cdot 10^{5}$ & 1.0 & $7.7 \cdot 10^{-9}$ \\
\hline
\end{tabular}

$(\mu \tau)_{\text {eff }}$ suitably combines the $\mu \tau$-products of electrons and holes $^{1}$, as resulting from the integration of (2).

Introducing this recombination current into the equivalent circuit (dashed symbol in Fig. 1) leads to the following analytical expression for the $I(V)$ curve of amorphous silicon solar cells and modules

$$
\begin{aligned}
I(V)= & -I_{\mathrm{ph}}+I_{\mathrm{ph}} \cdot \frac{d_{i}^{2}}{(\mu \tau)_{\mathrm{eff}}\left[V_{\mathrm{bi}}-\left(V-I R_{s}\right)\right]} \\
& +I_{0}\left(e^{\left(V-I R_{s}\right) /(n k T / e)}-1\right)+\frac{V-I R_{s}}{R_{p}} .
\end{aligned}
$$

The photogenerated current $I_{\mathrm{ph}}$ is reduced by the loss currents due to recombination in the i-layer, by the diode with its saturation current $I_{0}$ and its quality factor $n$ ( $e$ denotes here the elementary charge, $k$ Boltzmanns' constant and $T$ the absolute temperature of the device), and by the parallel resistance $R_{p}$.

Note that we expect this model to be valid for small forward voltages only, where the crude assumptions mentioned above can be considered to be fulfilled. This is the case in the short circuit region [4] and we find from (5) that the slope $R_{\mathrm{sc}}$ is determined by the recombination term of the model

$$
R_{\mathrm{sc}}=I_{\mathrm{ph}}^{-1} \cdot \frac{(\mu \tau)_{\mathrm{eff}} V_{\mathrm{bi}}^{2}}{d_{i}^{2}} .
$$

Here the effect of $R_{s}$ and $R_{p}$ have been neglected, which is correct for intermediate illumination levels (see Section IV). The measurement of $R_{\mathrm{sc}}$ provides direct information about the effective $(\mu \tau)$ eff-product within the i-layer of the device. Numerical simulation of amorphous silicon solar cells using the program described in [8] with a standard defect model demonstrates that the recombination current within the i-layer is the loss current showing the strongest variation with the external voltage $V$ [9]. Other loss currents (for example those due to recombination at the interfaces between the intrinsic and the doped layers) were found to have a very weak dependence on the external voltage leading thus to a neglible influence on $R_{\mathrm{sc}}[9]$.

The built-in voltage in amorphous silicon solar cells was determined by [10] to be $V_{\mathrm{bi}}=0.9 \mathrm{~V}$ and we will use this value for the following calculations. Assuming that $V_{\mathrm{bi}}$ does not change during degradation, monitoring $R_{\mathrm{sc}}$ allows direct measurement of the degradation state of the i-layer of the device.

\footnotetext{
${ }^{1}$ Note that recent investigations on $a$-Si:H films lead to the empirical conclusion that the $\mu \tau$-products are approximately equal in the case of compensated or strongly degraded $a$-Si:H [7], i.e., $(\mu \tau)_{\text {eff }} \approx \mu_{n}^{0} \tau_{n}^{0} \approx \mu_{p}^{0} \tau_{p}^{0}$. If, in fact $\mu_{n}^{0} \tau_{n}^{0}=\mu_{p}^{0} \tau_{p}^{0}$ holds, a more precise calculation of recombination losses within the i-layer of the pin cell can be employed for our model and leads to a final result that is identical to that given above in (3) and (4).
} 
The experimental data shown in Fig. 3 have been fitted with the model using the parameters shown in Table I. In the short circuit region, we note a perfect matching of the $R_{\mathrm{sc}^{-}}$ data with the model for both the degraded and as-deposited cell for the whole range of illumination levels covering six orders of magnitude.

In the open circuit region, the model loses its validity as the underlying theoretical assumptions are no longer fulfilled. It is, therefore, surprising how well the open circuit voltage of the undegraded cell matches that of the model. Note thereby that the slight underestimation of $V_{\mathrm{oc}}$ by the model for the degraded cell is caused by the weight of the recombination current term in (5). The values of $R_{\mathrm{oc}}$ predicted by the model in (5) do not depend on the recombination current term but mainly on the diode term; they remain slightly underestimated.

The validity of the model can also be seen from well matching of the explicit $I(V)$ curves shown in Fig. 2.

\section{THE REgIMES OF ILLUMINATION LEVELS}

The model for amorphous silicon solar cells and modules presented in (5) allows five regimes of illumination levels to be distinguished. These are shown schematically in Fig. 4 and marked in Fig. 3:

Regime A: At the lowest illumination levels, the $I(V)$ curve of the solar cell is dominated by its parallel resistance $R_{p}$ leading to a linear $I(V)$ curve as sketched in Fig. 4. Both $R_{\mathrm{oc}}$ and $R_{\mathrm{sc}}$ are equal to the parallel resistance $R_{p}$. This regime is characterized by the independence of $R_{\mathrm{sc}}$ and $R_{\mathrm{oc}}$ from the illumination: such a behavior can be observed on the left side of Fig. 3(a) and (b). In this illumination regime, the low parallel resistance $R_{p}$ leads to a reduced open circuit voltage $V_{\text {oc }}$ [Fig. 3(c)] as well as to a fill factor $F F$ of 0.25 (Fig. $3(\mathrm{~d})$ ). The lower the value of $R_{p}$, the higher the illumination level has to be to assure that the performance of the device is not affected by $R_{p}$. The detection of the parallel resistance in this illumination regime may be used for the quality control of commercial amorphous silicon solar modules.

Regime B: Increasing the illumination level, we reach regime $\mathbf{B}$ where the parallel resistance $R_{p}$ only dominates the short circuit part of the $I(V)$ curve as shown in Fig. 4. The fill factor $F F$ is still reduced by $R_{p}$ but the open circuit voltage $V_{\mathrm{oc}}$ is no more affected [Fig. 3(c) and (d)]. The inverse slope $R_{\mathrm{sc}}$ is still equal to the parallel resistance $R_{p}$, whereas $R_{\mathrm{oc}}$ is determined by the physics of the junction.

Regime C: In this regime, neither the series nor the parallel resistance $R_{p}$ affects the $I(V)$ curve of the device (Fig. $4)$. Both the slopes $R_{\mathrm{oc}}$ and $R_{\mathrm{sc}}$ are determined by the physics of the pin junction, and $R_{\mathrm{sc}}$ may be used to asses the recombination in the i-layer (6). In this regime, the fill factor $F F$ exhibits the best values; a fact which makes the regime $\mathbf{C}$ recommendable for the practical operation of amorphous silicon solar cells or modules. This is the only regime where the fill factor $F F$ is a correctly indicates the state of degradation of the device.

Regime D: Increasing the illumination level, we reach regime $\mathbf{D}$ where the series resistance $R_{s}$ of the device begins to affect the open circuit region of the $I(V)$ curve as indicated

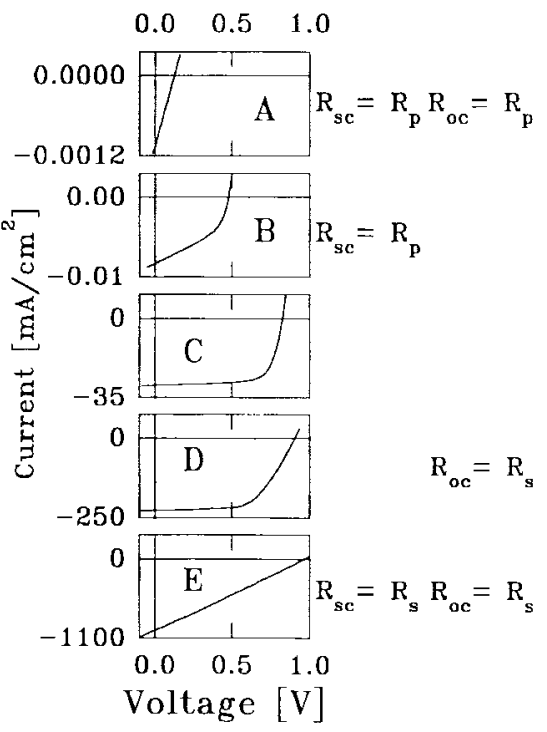

Fig. 4. The regimes of illumination levels. The five regimes of illumination levels for amorphous silicon solar cells and modules resulting from the model underlying equation (5). $R_{\mathrm{OC}}$ reflects the physics of the pin junction in the regimes $\mathbf{B}$ and $\mathbf{C}$. In the regimes $\mathbf{C}$ and $\mathbf{D}, R_{\mathrm{sc}}$ is determined by the recombination in the i-layer (6).

schematically in Fig. 4. Thereby, the open circuit voltage $V_{\mathrm{OC}}$ is unaffected; however, the open cicuit resistance $R_{\mathrm{oc}}$ is now determined by the series resistance $R_{s}$. This leads to a reduced fill factor $F F$. The short circuit region, and especially $R_{\mathrm{sc}}$ are not affected by $R_{s}$. This regime is not reached by the laboratory cell shown in Fig. 3 because of its low series resistance, but it is reached by commercial modules under one sun illumination. It is characterized by the flattening of the dependence of $R_{\mathrm{oc}}$ with the illumination level. This is shown in Fig. 5 for a commercial module, where the illumination level is presented by the open circuit voltage.

Regime E: At the highest illumination levels we reach regime $\mathbf{E}$, where the $I(V)$ curve predicted by the model is completly dominated by the series resistance $R_{s}$ leading to a linear $I(V)$ curve with a fill factor $F F$ of 0.25 (Fig. 4). In this regime the inverse slopes $R_{\mathrm{oc}}$ and $R_{\mathrm{sc}}$ are equal to the series resistance $R_{s}$ of the device.

\section{Evaluation of ThE PARAmeters}

The evaluation of the elements of the equivalent circuit shown in Fig. 1 and described by the analytical expression of (5) is based on the variable illumination measurement (VIM) of the $I(V)$ curve indicated in Section II, and a subsequent presentation of their parameters as shown in Fig. 3.

The parameters of the diode term are determined from the $V_{\mathrm{oc}}\left(I_{\mathrm{sc}}\right)$-behavior of the device. For the intermediate illumination regime $\mathbf{C}$ where the effects of the series and parallel resistance $\left(R_{s}, R_{p}\right)$ can be neglected, the analytical model (5) yields the following expression:

$$
V_{\mathrm{oc}}=\frac{n k T}{e} \ln \left(\frac{I_{\mathrm{sc}}}{I_{0}}\right) .
$$

Here, we have also neglected the effect of the recombination losses in the i-layer. Fitting this equation to the logarithmic 
region of the measured $V_{\mathrm{oc}}\left(I_{\mathrm{sc}}\right)$-behavior [Fig. 3(a)] yields the diode saturation current $I_{0}$ and the quality factor $n$.

The high illumination limit of $R_{\mathrm{Oc}}$ is, according to the model, equal to the series resistance $R_{S}$, which may be taken as the asymptotic saturation value of $R_{\mathrm{OC}}$ (regimes $\mathbf{D}$ and $\mathbf{E}$ ). The series resistance $R_{s}$ is physically determined by the sheet resistance of the electrodes, especially that of the transparent electrode.

The low illumination limit of $R_{\mathrm{sc}}$ is, according to the model, equal to the parallel resistance $R_{p}$, which may be taken as the asymptotic saturation value of $R_{\mathrm{sc}}$ (regimes $\mathbf{B}$ and $\mathbf{A}$ ). $R_{p}$ may also be determined by the saturation of the slope $\left(\partial V / \partial I_{\text {dark }}\right)$ for low dark currents $I_{\text {dark }}$ (see dotted line in Fig. 3(b), note that low dark currents-here less than $3 \cdot 10^{-4} \mathrm{~mA} / \mathrm{cm}^{2}$-are determined by $R_{p}$ and not by the pin-junction). The parallel resistance $R_{p}$ is a measure of the technological quality of the device: low values of $R_{p}$ indicate high leakage currents due to pin-holes.

The product $(\mu \tau)_{\text {eff }}$, is the key parameter which characterizes the new recombination term in the model; are determined from the power-law regime of the experimental $R_{\mathrm{sc}}\left(I_{\mathrm{sc}}\right)$-data [regime C, see Fig. 3(b)] using (6). The built-in voltage $V_{\mathrm{bi}}$ can be assumed to be approximately $0.9 \mathrm{~V}$ (for a more precise but rather cumbersome experimental determination of $V_{\mathbf{b i}}$ see [11] or [10]).

This evaluation procedure, when applied to the typical laboratory cell described in Section II, yields the parameters shown in Table I. Using these parameters with the model in equation (5) to calculate $V_{\mathrm{oc}}, F F, R_{\mathrm{sc}}$ and $R_{\mathrm{oc}}$, yields the lines shown in Fig. 3; note their excellent fit with the experimental data. This signifies that the new equivalent circuit in Fig. 1 and the analytical model in equation (5) describe quite satisfactorily the full behavior of the solar cell tested, over the whole range of six orders of illumination levels.

\section{Application: Splitting the Degradation EfFects}

One key feature of the VIM-method is the measurement of the slopes $R_{\mathrm{oc}}$ and $R_{\mathrm{sc}}$. We can now use these slopes to determine the reasons for the degradation of a commercial amorphous silicon module during outdoor exposition.

On one side, the analytical model in (5) results in $R_{\mathrm{oc}}$ being equal to the series resistance $R_{s}$ of the device in the high illumination limit (regime D). At one-sun illumination, this limit is not reached by the small-area laboratory cell whose data are shown in Fig. 3(a), but it is reached by commercial, large-area modules with their higher series resistance $R_{s}$. This is shown in Fig. 5, where the clear saturation of $R_{\mathrm{oc}}$ allows the series resistance of the module to be determined. The $R_{\mathrm{OC}^{-}}$ data shown here were obtained by scanning the $I(V)$ curve in ten-minutes intervals during outdoor exposition. By filtering these $R_{\mathrm{oc}}$-data for high illumination conditions, they directly present the series resistance $R_{s}$. Fig. 6(a) shows the continuous increase of $R_{s}$, which has to be related to degradation effects outside the pin-junction, for example degradation of the $\mathrm{SnO}_{2}$ layer or corrosion of the contacts. It should be mentioned here that several other commercial amorphous silicon modules examined did not show such a degradation of $R_{s}$.

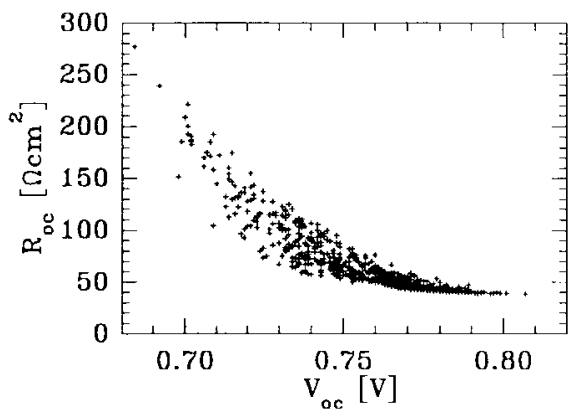

Fig. 5. $\quad R_{\mathrm{OC}}\left(V_{\mathrm{OC}}\right)$-data of a commercial module obtained by scanning the $I(V)$ curve in ten-minute intervalls from day 184 to 202 of outdoor exposition. These data are temperature-corrected using measured temperature coefficients of $R_{\mathrm{OC}}$ and $V_{\mathrm{OC}}$

(a)

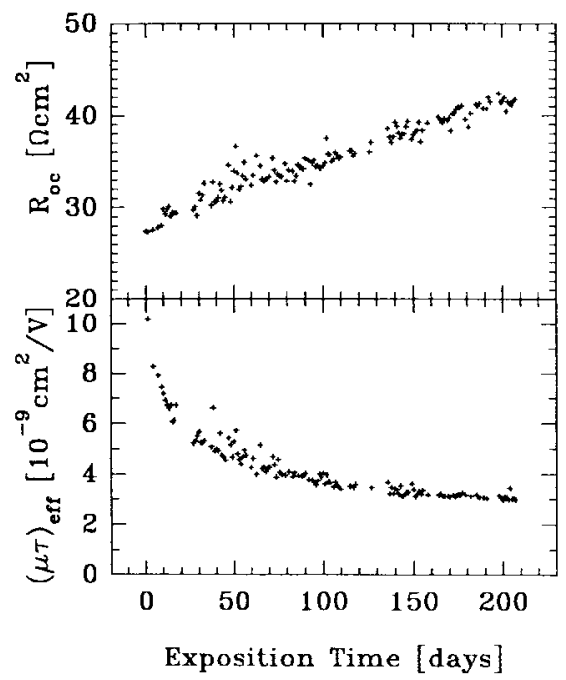

Fig. 6. $\quad R_{\mathrm{OC}}$ and $(\mu \tau)_{\text {eff }}$-data of a commercial module. They are filtered for clear irradiance conditions around solar noon and then daily averaged. $(\mu \tau)_{\text {eff }}$ has been calulated from $R_{\mathrm{sc}}$ assuming $d_{i}=0.3 \mu \mathrm{m}$ and a constant $V_{\mathrm{bi}}=0.9 \mathrm{~V}$. These data are temperature-corrected using measured temperature coefficients of $R_{\mathrm{OC}}$ and $R_{\mathrm{sc}}$.

On the other side, the inherent light-induced degradation of the i-layer material (Staebler-Wronski effect [12]) of the module is determined by the effective $(\mu \tau)_{\text {eff-product calcu- }}$ lated from $R_{\mathrm{sc}}$ with (6). Fig. 6(b) shows the data for the same module assumnig a constant built-in voltage during outdoor exposure. The apparent tendency to saturate may be caused by a slight seasonal effect, as the data shown have been monitored until the beginning of Summer 1995.

The slope $R_{\mathrm{sc}}$ is equal to the parallel resistance $R_{p}$ in the low illumination limit according to the model in (5); it is detected by the flattening of the $R_{\mathrm{sc}}\left(I_{\mathrm{sc}}\right)$-data which is shown for the laboratory cell in Fig. 3(b). The parallel resistance $R_{p}$ is slightly reduced in the degraded cell. We attribute this decrease to the formation of pin-holes during degradation; such pin-holes may have been created by the mechanical stress provoked by the contact finger during the degradation period. Mechanical stress is known to reduce the parallel resistance $R_{p}$ of amorphous silicon solar cells, a behavior which frequently occurs when the cells are mechanically cut [13].

At higher illumination levels $\left[I_{\mathrm{sc}}>0.1 \mathrm{~mA} / \mathrm{cm}^{2}\right.$ in Fig. $3(b)]$, the reduction of the parallel resistance does not 
affect the performance of the degraded cell. Now the slope $R_{\mathrm{sc}}$ is determined by the reduced effective $\mu \tau$-product $(\mu \tau)_{\text {eff }}$ due to the light-induced defect creation in the i-layer.

Within the framework of the model given by (5), the enhanced recombination in the i-layer however does not explain the loss of open circuit voltage of the degraded cell shown in Fig. 3(d). This degradation effect is taken into account with an increase of the diode blocking current $I_{s}$ by nearly two orders of magnitude (see Table I). This increase of the diode term in the model represents enhanced recombination in the regions of the $\mathrm{p} / \mathrm{i}$ and $\mathrm{n} / \mathrm{i}$ interface and other degradation effects linked to the semiconductor barriers. This shows that the VIM-data permit to distinguish bulk i-layer degradation from other degradation effects inside the pin-structure of the device.

\section{CONCLUSIONS}

An improved equivalent circuit for amorphous silicon solar cells and modules has been presented. The related analytical model for these devices is simple and was shown to match the current-voltage $I(V)$ curves of a typical laboratory amorphous silicon cell for a wide range of illumination levels varying over six orders of magnitude. The model is a single exponential model with a new term taking into account the recombination in the i-layer of the device. Combined with the experimental method of Variable Illumination Measurement (VIM) of the $I(V)$ curve (an experimental method which can also be used outdoors under sunlight), this model allows for a precise determination of the series and parallel resistances and for a clear discrimination between different degradation effects. So is the reciprocal slope $R_{\mathrm{sc}}=$ $(\partial V / \partial I)_{V=0}$ of the $I(V)$ curve at the short circuit point, at intermediate illumination levels, directly proportional to the effective $\mu \tau$-product $(\mu \tau)_{\text {eff }}$ in the i-layer and thus serves as a quantifying tool for the state of degradation of the cell or module.

Based on the measurements of a commercial module, we have demonstrated how the measurement of the series resistance $R_{s}$ and of the effective $\mu \tau$-product $(\mu \tau)_{\text {eff }}$ in the i-layer leads to a discrimination between two different degradation effects occuring in a commercial amorphous silicon module: in this case the Staebler-Wronski degradation effect has been shown to flatten after 200 days of outdoor exposition, whereas the series resistance $R_{s}$ has been shown to be linked to an additional deterioration effect not showing any tendency to saturate.

For a typical laboratory cell, it could be clearly demonstrated that its light-induced degradation cannot be exclusivley attributed to an enhanced recombination in the i-layer of the device.

\section{ACKNOWLEDGMENT}

The authors would like to thank A. Muñoz for assistance during the VIM measurements and to $\mathrm{C}$. Hof for revising the manuscript.

\section{REFERENCES}

[1] M. A. Green, Solar Cells-Operating Principles, Technology and System Application. Univ. of New South Wales, Kensington, 1992.

[2] H. G.Wagemann and H. Estrich, Grundlagen der photovoltaischen Energiewandlung. Stuttgart, Germany: Teubner, 1994.

[3] W. Kusian, H. Pfleiderer, and W. Jürgens, "Buffer layer and light degradation of $a$-Si pin solar cells," in Proc. 9th E.C. Photovoltaic Solar Energy Conf., 1989, pp. 52-55.

[4] J. Hubin and A. V. Shah, "Effect of recombination function on the collection in a p-i-n solar cell," Philos. Mag. B, vol. 72, no. 6, pp. 589-599, 1995.

[5] R. Platz, D. Fischer, C. Hof, S. Dubail, J. Meier, U. Kroll, and A. Shah, " $\mathrm{H}_{2}$-dilution versus buffer layers for increased $V_{\mathrm{oc}}$," in Proc. Mater. Res. Soc. Symp., 1996, vol. 420, pp. 51-56.

[6] J. Hubin, A. V. Shah, and E. Sauvain, "Effects of dangling bonds on the recombination function in amorphous semiconductors," Philos. Mag. Lett., vol. 66, pp. 115-125, 1992.

[7] N. Beck, N. Wyrsch, C. Hof, and A. V. Shah, "Mobility lifetime product $-\mathrm{a}$ tool for correlating $a$-Si:H film properties and solar cell performance," J. Appl. Phys., vol. 79, pp. 9361-9367, 1996.

[8] J. M. Asensi, J. Andreu, J. Puigdollers, and J. Bertomeu, "Interface effects in amorphous silicon solar cells," in Proc. 11th E.C. Photovoltaic Solar Energy Conf., 1992, pp. 769-772.

[9] J. Merten, Photovoltaics with Amorphous Silicon: Technological, Physical and Technical Aspects, Ph.D. dissertation, Universitat de Barcelona, Barcelona, Spain, 1996.

[10] S. Nonomura, Y. Hamakawa, and H. Okamoto, "Determination of the built-in potential in $a$-Si solar-cells by means of electro-absorption method," Jpn. J. Appl. Phys.: Part II-Letters, vol. 21, pp. L464-L466, 1982.

[11] L. Jiang, Q. Wang, E. Schiff, S. Guha, Y. Yang, and X. Ding, "Electro absorption measurements and built-in potentials in amorphous silicon pin solar cells," submitted for publication.

[12] D. L. Staebler and C. R. Wronski, "Reversible conductivity changes in discharge-produced amorphous silicon," Appl. Phys. Lett., vol. 31, pp. 292-294, 1977.

[13] T. Eickhoff, H. Striebig, W. Raetz, B. Rech, and H. Wagner, "Temperature dependence of $a$-Si:h solar cell prameters-Experiment and numerical simulations," in Proc. 13th Int. Photovoltaic Science and Engineering Conf., 1995, p. 238.

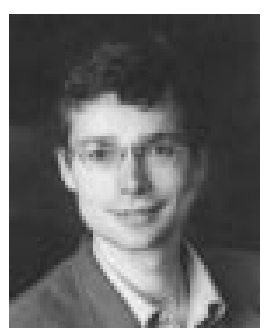

J. Merten was born in Freiburg, Germany, in 1965. He received the diploma degree of technical physics at Munich Technical University, Munich, Germany, in 1993. He then studied the degradation of amorphous silicon films at the Thin Films Lab at École Polytechnique, France, and received the Ph.D. degre from Barcelona University, Barcelona, Spain, in 1996, were his dissertation covered technological, physical, and application aspects of amorphous silicon photovoltaics. On the technological side, he worked on the optimization of the production process of amorphous silicon solar cells, including contamination aspects. On the application side, he designed outdoor measurement systems to monitor amorphous silicon photovoltaic devices on cell, module, and generator level.

Since 1996, he has been a Professor at the Salesian University School in Sarriá, Barcelona, where his main interest is in photovoltaics and instrumentation.

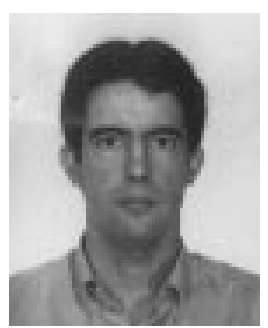

J. M. Asensi was born in Barcelona, Spain, on May 20, 1964. He received the B.Sc. degree in 1987 and the Ph.D. degree in physics in 1994, both from Barcelona University. His doctoral thesis covered deposition, characterization, and physics modeling of amorphous silicon thin-film solar cells.

Currently, he is Assistant Professor for Applied Physics at Barcelona University, where his research interests include amorphous silicon physics and computer modeling of solar cells. 


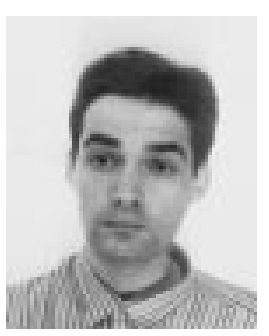

C. Voz was born in Barcelona, Spain, on November 13, 1972. He received the degree in physics from the University of Barcelona in 1995, where he is currently pursuing the Ph.D. degree in the Applied Physics and Electronics Department. His research activities cover optical and electrical properties of amorphous and polycrystalline silicon, as well as the related material and device technology.

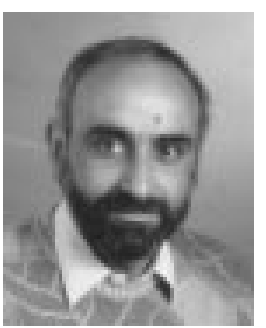

A. V. Shah was born in Mumbai (formerly Bombay), India, in 1940. He was educated in London, Zürich, and Bombay. He received the Dipl. El. Ing. degree in 1964, and the Ph.D. degree in 1969, both from ETH Zürich.

Since 1979, he has been Professor at the University of Neuchâtel, Switzerland, and has also been a part-time Professor at the EPF Lausanne since 1988. He founded the Centre for Electronics Design and Technology (CEDT) at the Indian Institute of Science in 1975. His main professional interest is

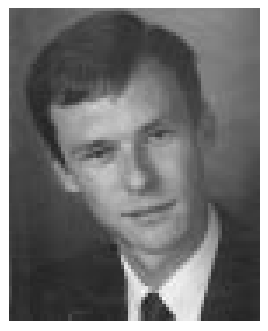

R. Platz was born in Villigen, Germany, in 1967. He received the degree in electrical engineering from the University of Stuttgart, Stuttgart, Germany, in 1993.

In 1994, he joined the group of Prof. A. V. Shah at the Institute of Microtechnology of the University of Neuchâtel, Switzerland, where he is currently pursuing the Ph.D. degree in the field of thin-film amorphous silicon solar cells with a special emphasis on the stability of amorphous silicon solar cells.

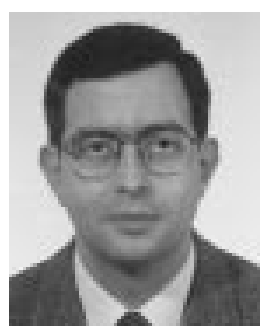

J. Andreu was born in Barcelona, Spain, in 1958. He received the degreein physics in 1980, and the $\mathrm{Ph} . \mathrm{D}$. degree in physics and technology of materials in 1986, both from the University of Barcelona.

In 1988, he became Professor at the Applied Physics Department of the University of Barcelona, where, since 1989, he has been heading the photovoltaic group. His main professional interest is thin-film solar cells technology. 